\title{
Diallyl disulfide attenuates non-alcoholic steatohepatitis by suppressing key regulators of lipid metabolism, lipid peroxidation and inflammation in mice
}

\author{
NING ZHANG $^{1 *}$, YULI WANG ${ }^{2 *}$, JUNLI ZHANG $^{1}$, BEIBEI LIU ${ }^{1}$, \\ GUIXIN LI ${ }^{1}$, SHENGLIANG XIN ${ }^{1}$ and KESHU XU ${ }^{1}$ \\ ${ }^{1}$ Division of Gastroenterology, Union Hospital, Tongji Medical College, \\ Huazhong University of Science and Technology, Wuhan, Hubei 430022; ${ }^{2}$ Department of Oncology, \\ The First Affiliated Hospital of Medical College, Shihezi University, Shihezi, Xinjiang 832002, P.R. China
}

Received November 2, 2018; Accepted May 3, 2019

DOI: $10.3892 / \mathrm{mmr} .2019 .10316$

\begin{abstract}
Non-alcoholic steatohepatitis (NASH) is a common clinicopathological condition. Currently, the pathogenesis of NASH remains unknown, and no optimal therapy option currently exists. It has previously been demonstrated that diallyl disulfide (DADS) was capable of attenuating liver dysfunction, as DADS supplementation had a positive impact on liver regeneration, proliferation and oxidative damage. Thus, DADS could serve as a potential therapeutic agent that can protect against the effects of NASH. The present study aimed to evaluate the effect of DADS on NASH and to understand the associated underlying molecular mechanisms. A methionine- and choline-deficient diet (MCD) and high-fat diet (HFD) are the two common animal models that induce NASH. C57BL/6J mice were fed an MCD for 4 weeks, or an HFD for 20 weeks, in the present study. The mice were treated with or without DADS $(20,50$ and $100 \mathrm{mg} / \mathrm{kg}$ ) for 4 or 20 weeks. For the histopathological examination, hematoxylin and eosin staining, oil red $\mathrm{O}$ staining and immunohistochemical analyses were performed using the liver sections. Biochemical assays and ELISA were performed to measure the serum biochemical indicators of hepatic function and inflammatory indicators, respectively. Reverse transcription-quantitative PCR, immunofluorescence and western blotting were used to detect expression levels of the genes involved in the molecular mechanisms underlying DADS protection. MCD or HFD
\end{abstract}

Correspondence to: Dr Keshu Xu, Division of Gastroenterology, Union Hospital, Tongji Medical College, Huazhong University of Science and Technology, 1277 Jiefang Avenue, Wuhan, Hubei 430022, P.R. China

E-mail: xuzoumedmail@126.com

${ }^{*}$ Contributed equally

Key words: diallyl disulfide, non-alcoholic steatohepatitis, lipid metabolism, lipid peroxidation, inflammation induced the histological features of NASH in mice, including significant vacuolated hepatocytes, marked inflammatory cell infiltration and severe micro- and macro-vesicular steatosis. Serum alanine transferase and aspartate aminotransferase levels, as well as the contents of liver triglyceride and total cholesterol, were significantly increased in these two models. DADS attenuated these histological and biochemical changes. DADS ameliorated hepatic steatosis by regulating sterol regulatory element-binding transcription factor 1, apolipoprotein A1, cyclic AMP-responsive element-binding protein $\mathrm{H}$ and fibroblast growth factor 21. Furthermore, DADS was revealed to prevent lipotoxicity via peroxisome proliferator-activated receptor $\alpha$ elevation and stearoyl-coenzyme A desaturase 1 inhibition in HFD-fed mice. In addition, DADS markedly inhibited lipid peroxidation by modulating malondialdehyde and superoxide dismutase, and it also decreased tumor necrosis factor- $\alpha$ production, interleukin- 6 production and macrophage influx, as well as suppressing nuclear factor- $\kappa \mathrm{B}$ activation, indicating suppression of MCD-induced hepatic inflammation. Taken together, the results have shown that DADS exerts beneficial effects on MCD- or HFD-induced NASH by suppressing key regulators of lipid metabolism, lipid peroxidation and inflammation.

\section{Introduction}

Nonalcoholic steatohepatitis (NASH) is a common clinicopathological disease characterized by lipid deposition, inflammatory lesions and hepatocyte damage, which may induce varying degrees of liver cirrhosis or result in the development of hepatocellular carcinoma (1). Previous evidence has demonstrated that NASH can be induced by several factors, such as lipotoxicity, oxidative stress, genetic susceptibility, mitochondrial dysfunction, abnormal lipid metabolism and endoplasmic reticulum stress $(2,3)$. Currently, the pathogenesis of NASH remains unclear, and key events in NASH have always been targets of therapeutic management (4). Thus, physical exercise, weight loss and lifestyle adjustments remain the best treatment methods for NASH. Furthermore, the absence of approved pharmacological 
therapies has contributed to expanse of extensive research into NASH treatment.

Garlic compounds have gained increasing attention over the last few decades, owing to their diverse biological activities, including their antioxidant, antiatherosclerotic, antithrombotic, antidiabetic, anticarcinogenic and anti-inflammatory effects (5-7). Diallyl disulfide (DADS), a major component of the secondary organosulfur compounds derived from garlic, has demonstrated protective effects against cancer development, as well as against the onset of allergies, arthritis, and cardiovascular, neurological, and liver diseases (8-11). Drug metabolism can be divided into one of two phases: The first phase is called a biotransformation reaction, which includes oxidation, reduction and hydrolysis reactions; and the second phase is known as the binding reaction, where the reaction is combined with endogenous substances. These beneficial effects may be attributed to DADS-mediating phase I and II metabolizing enzymes $(12,13)$. DADS was demonstrated to inhibit dichloroacetate-induced reactive oxygen species (ROS) production, inflammatory factors and the expression of p50 in the nucleus in a dose-dependent manner. Feng et al (14) revealed that DADS may be a good candidate for the chemical prevention and therapy of Barrett's esophagus and esophageal adenocarcinoma. In addition, DADS has previously been reported to induce mitochondrial biogenesis to attenuate cardiac hypertrophy via the eNOS-Nrf2-Tfam pathway in rats (15). Previous studies have also suggested that the use of DADS supplementation following major hepatectomy had a positive impact on liver regeneration, proliferation and oxidative damage $(16,17)$. DADS was capable of attenuating liver dysfunction, as it had been metabolized into allylglutathione sulfide and allylmercaptan in rats and humans (18). However, the effects of DADS in NASH, and the associated underlying molecular mechanisms, remain unclear.

In the present study, the effect of DADS on NASH models induced by a methionine- and choline-deficient diet (MCD) or a high-fat diet (HFD) was evaluated. The potential mechanisms of the protective effects of DADS, in regard to lipid metabolism, lipid peroxidation, lipotoxicity and inflammation, were also further investigated.

\section{Materials and methods}

Animals and experimental groups. The experimental protocol was approved by the Animal Ethics Committee of Tongji Medical College, Huazhong University of Science and Technology (Wuhan, China), and performed according to the principles of the Institutional Animal Care and Ethics Committee guidelines (IACUC no. S619). A total of 60 male C57BL/6J mice weighing $22 \mathrm{~g}$ (6-7 weeks of age) were purchased from Beijing Huafukang Bioscience Co., Ltd., and maintained in a temperature-controlled room $\left(23 \pm 2^{\circ} \mathrm{C}\right)$ with a 12-h light-dark lighting cycle.

NASH mice models were established via an MCD for 4 weeks and an HFD for 20 weeks (19). These models demonstrated increased adipose tissue inflammation, enlarged fat cells and less oxidized fat in their muscles. DADS (purity $>75 \%$; Sigma-Aldrich; Merck KGaA) dissolved in corn oil was prepared immediately prior to treatment. Following a 2-week acclimation period with standard pellet chow and water ad libitum given prior to the experiments, the mice were randomly divided into one of the following 12 groups ( $n=5$ mice per group): i) Mice fed a normal diet with daily oral gavage of corn oil [Normal + negative control (NC) group]; ii) mice fed a normal diet with daily oral gavage of $20 \mathrm{mg} / \mathrm{kg}$ DADS (Normal + DADS-L group); iii) mice fed a normal diet with daily oral gavage of $50 \mathrm{mg} / \mathrm{kg}$ DADS (Normal + DADS-M group); iv) mice fed a normal diet with daily oral gavage of $100 \mathrm{mg} / \mathrm{kg}$ DADS (Normal + DADS-H group); v) mice fed an MCD diet with daily oral gavage of corn oil (MCD + NC group); vi) mice fed an MCD diet with daily oral gavage of $20 \mathrm{mg} / \mathrm{kg}$ DADS (MCD + DADS-L group); vii) mice fed an MCD diet with daily oral gavage of $50 \mathrm{mg} / \mathrm{kg}$ DADS (MCD + DADS-M group); viii) mice fed an MCD diet with daily oral gavage of $100 \mathrm{mg} / \mathrm{kg}$ DADS (MCD + DADS-H group); ix) mice fed an HFD diet with daily oral gavage of corn oil (HFD + NC group); $\mathrm{x}$ ) mice fed an HFD diet with daily oral gavage of $20 \mathrm{mg} / \mathrm{kg}$ DADS (HFD + DADS-L group); xi) mice fed an HFD diet with daily oral gavage of $50 \mathrm{mg} / \mathrm{kg}$ DADS (HFD + DADS-M group); and xii) mice fed an HFD diet with daily oral gavage of $100 \mathrm{mg} / \mathrm{kg}$ DADS (HFD + DADS-H group). The DADS concentrations were selected based on previous studies with minor modifications $(5,17)$.

Following treatment with MCD for 4 weeks and HFD for 20 weeks, the mice were sacrificed following a 12-h fast via pentobarbital sodium injection. Blood samples were collected from the heart following euthanasia and serum was isolated via centrifugation at $4^{\circ} \mathrm{C}$ for $15 \mathrm{~min}$ at $2,000 \mathrm{x}$ g. Liver tissues were collected and weighed following washing with physiological saline, and then a portion of the tissue was fixed with $4 \%$ paraformaldehyde for $24 \mathrm{~h}$ at room temperature to prepare for histological staining. The remaining hepatic tissues were stored at $-80^{\circ} \mathrm{C}$ for molecular biological assays.

Liver histology and immunohistochemical staining of F4/80-positive cells. Paraformaldehyde-fixed liver tissues were used for hematoxylin and eosin (H\&E) staining and oil red $O$ staining, as described in a previous study (20). The NASH activity score, a histological feature scoring system, was utilized to assess the liver lesions: Steatosis (score, 0-3), lobular inflammation (score, 0-3), and hepatocellular ballooning (score, 0-2). Samples with scores $>4$ were diagnosed as 'NASH', samples with scores $<3$ were designated as 'not NASH', and samples with scores between 3-4 were designated as 'possible for NASH' (21). The expression of F4/80, a macrophage-surface marker, was detected via immunohistochemistry using an immunohistochemistry kit (Wuhan Boster Biological Technology, Ltd.) with the rat anti-F4/80 monoclonal antibody (1:3,000; cat. no. MCA497; Bio-Rad Laboratories, Inc.) at $37^{\circ} \mathrm{C}$ for $2 \mathrm{~h}$, and subsequent incubation at $37^{\circ} \mathrm{C}$ for $2 \mathrm{~h}$ with the secondary antibodies (horseradish peroxidase-conjugated anti-mouse $\mathrm{IgG} ; 1: 2,000$; cat. no. SV0004; Wuhan Boster Biological Technology, Ltd.). Detection of positive staining was performed using 3,3'-diaminobenzidine reagent (Wuhan Boster Biological Technology, Ltd.) for $3 \mathrm{~min}$ at room temperature. Finally, all sections were counterstained with $10 \%$ hematoxylin for $30 \mathrm{sec}$ at room temperature, and were then left to dry and covered with a coverslip. For every section, 5 fields were randomly selected to evaluate the quantification of F4/80 
Table I. Primer sequences for reverse transcription-quantitative PCR assays.

\begin{tabular}{lll} 
Genes & \multicolumn{1}{c}{ Forward primers $\left(5^{\prime}-3^{\prime}\right)$} & Reverse primers $\left(5^{\prime}-3^{\prime}\right)$ \\
\hline SREBP-1c & GCAGCCACCATCTAGCCTG & CAGCAGTGAGTCTGCCTTGAT \\
ApoA-1 & GCTCAAGAGCAACCCTACCTT & GCTTTCTCGCCAAGTGTCTTC \\
CREBH & CCTCCCGCTTCAACCTCAC & CCGGATCTTTCTGCGGATTTT \\
FGF2 1 & GTGTCAAAGCCTCTAGGTTTCTT & GGTACACATTGTAACCGTCCTC \\
PPAR $\alpha$ & GTCATCACAGACACCCTCTCTCC & TGTCCCCACATATTCGACACTC \\
SCD-1 & CAGTTCCTACACGACCACCACTA & GGACGGATGTCTTCTTCCAGAT \\
$\beta$-actin & TTCTTTGCAGCTCCTTCGTTGCCG & TGGATGGCTACGTACATGGCTGGG
\end{tabular}

using Image-Pro Plus 6.0 (Media Cybernetics, Inc.) under a light microscope (magnification, $\mathrm{x} 40$ ).

Lipid and transaminase analysis. Serum aspartate aminotransferase (AST) and alanine aminotransferase (ALT) levels were measured using biochemical methods according to protocols by the Nanjing Jiancheng Bioengineering Institute. Serum triglyceride (TG) and total cholesterol (TC) levels were measured using GPO-PAP and COD-PAP enzymatic colorimetric assay kits (Nanjing Jiancheng Bioengineering Institute), respectively, according to the manufacturer's protocol.

Cytokine measurement. The plasma concentrations of malondialdehyde (MDA), superoxide dismutase (SOD), interleukin (IL)- 6 and tumor necrosis factor (TNF)- $\alpha$ were measured using specific ELISA kits (BioLegend, Inc.) according to the manufacturer's protocol. The absorbance of the samples and standards was detected at 450 and $570 \mathrm{~nm}$ for the respective kits using a microplate reader (Bio-Rad Laboratories, Inc.).

Immunofluorescence. Paraffin-embedded liver sections (4- $\mu \mathrm{m}$ thickness) were dewaxed with dimethyl-benzene, permeabilized with $0.3 \%$ Triton X-100, and blocked with normal donkey serum (GeneTex, Inc.) for $60 \mathrm{~min}$ at room temperature. The sections were then co-incubated with cyclic AMP-responsive element-binding protein $\mathrm{H}(\mathrm{CREBH})$ antibody (1:100; cat. no. sc-377332; Santa Cruz Biotechnology, Inc.), and peroxisome proliferator-activated receptor $\alpha(\operatorname{PPAR} \alpha)$ antibody (1:100; cat. no. ab8934; Abcam) or stearoyl-coenzyme A desaturase 1 (SCD-1) antibody (1:100; cat. no. 2794; Cell Signaling Technology, Inc.) overnight at $4^{\circ} \mathrm{C}$. After being washed with phosphate buffer, the samples were incubated with secondary antibodies, including Alexa Fluor ${ }^{\circledR}$ 488-linked (green) anti-mouse and Alexa Fluor ${ }^{\circledR}$ 647-linked (red) anti-rabbit antibodies (1:200; cat. nos. R37114 and A-31573; both from Thermo Fisher Scientific, Inc.). Nuclei were stained using DAPI at room temperature for $10 \mathrm{~min}$. All slides were examined under a fluorescent confocal microscope (magnification, x40; FV-500; Olympus Corporation). Images were acquired using simultaneous dual-channel scanning.

Reverse transcription-quantitative $(R T-q) P C R$. Total RNA from the liver tissues was extracted using TRIzol ${ }^{\circledR}$ reagent (Thermo Fisher Scientific, Inc.). Next, $5 \mu \mathrm{g}$ of RNA was used for cDNA synthesis using an M-MLV RTase cDNA Synthesis kit (Takara Bio Inc.). Then, RT-qPCR was performed using a SYBR Green PCR kit (Takara Bio Inc.) with the LightCycler480 sequence detector system (Roche Applied Science), using the following reaction conditions: $95^{\circ} \mathrm{C}$ for $5 \mathrm{~min}, 45$ cycles of $95^{\circ} \mathrm{C}$ for $10 \mathrm{sec}, 60^{\circ} \mathrm{C}$ for $20 \mathrm{sec}$ and $70^{\circ} \mathrm{C}$ for $30 \mathrm{sec}$. The primer sequences for the RT-qPCR are listed in Table I. The $2^{-\Delta \Delta \mathrm{Cq}}$ method was used to calculate the relative mRNA expression (22), which was normalized against the internal control gene, $\beta$-actin. Gene expression levels for each sample were analyzed in duplicate.

Western blot assay. Tissue lysates were prepared using RIPA lysis buffer containing $0.1 \mathrm{mmol} / 1$ phenylmethylsulphonyl fluoride (PMSF; Sigma-Aldrich; Merck KGaA). After being quantified using a Bio-Rad protein assay kit (Bio-Rad Laboratories, Inc.), protein extracts (50 $\mu \mathrm{g} /$ lane) were separated via $10 \%$ SDS-PAGE and transferred onto a polyvinylidene fluoride (PVDF) membrane (EMD Millipore). Membranes were blocked with $5 \%$ fat-free milk at room temperature for $60 \mathrm{~min}$, followed by incubation with primary antibodies overnight at $4^{\circ} \mathrm{C}$. The membranes were washed and subsequently incubated at room temperature for $2 \mathrm{~h}$ in the next day with the secondary antibodies [horseradish peroxidase-conjugated anti-rabbit $\operatorname{IgG}(1: 2,000$; cat. no. GTX213110-01) and horseradish peroxidase-conjugated anti-mouse IgG (1:2,000; cat. no. GTX213111-01); both GeneTex, Inc.]. Immunoreactive proteins were visualized using chemiluminescence kits (Thermo Fisher Scientific, Inc.). The protein expression of each sample was analyzed three times and normalized against the internal reference antibody, GAPDH or tubulin.

The following primary antibodies were used: Anti-sterol regulatory element-binding transcription factor 1 (SREBP1c; 1:1,000; cat. no. ab28481; Abcam); anti-CREBH (1:1,000; cat. no. sc-377332; Santa Cruz Biotechnology, Inc); anti-PPAR $\alpha$ (1:2,000; cat. no. ab8934; Abcam); anti-SCD-1 (1:1,000; cat. no. 2794; Cell Signaling Technology, Inc.); anti-phospho (p)-nuclear factor (NF)-кB p65 (1:2,000; cat. no. 3033; Cell Signaling Technology, Inc.); anti-tubulin (1:2,000; cat. no. GTX76511; GeneTex, Inc.); and anti-GAPDH (1:2,000; cat. no. GTX100118; GeneTex, Inc.).

Statistical analysis. Values are expressed as the mean \pm standard error of the mean (SEM). All statistical analyses were performed using SPSS software (version 16.0; SPSS 
A

A 100
MCD

$00 \mathrm{mg}$ DADS $50 \mathrm{mg}$ DADS $20 \mathrm{mg}$ DADS

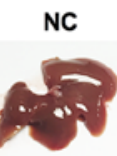

HFD

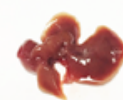

政

Normal
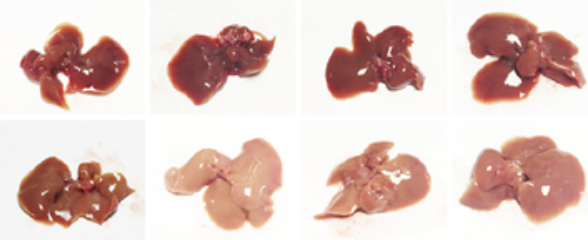

C

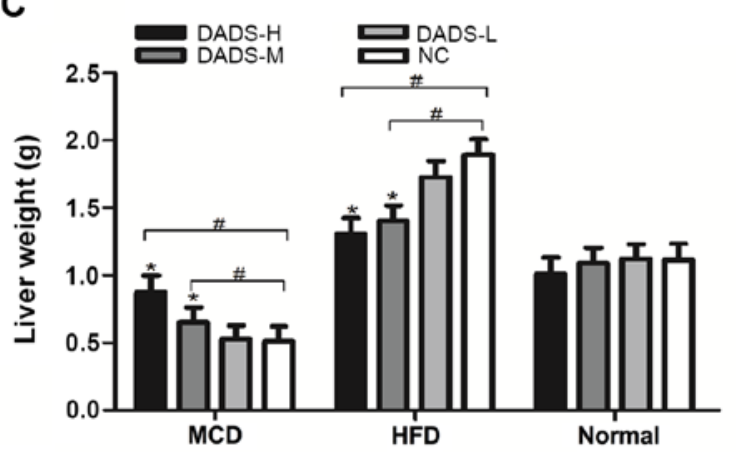

E

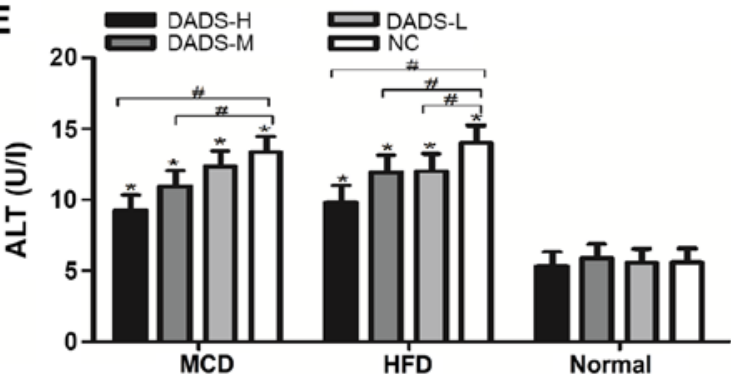

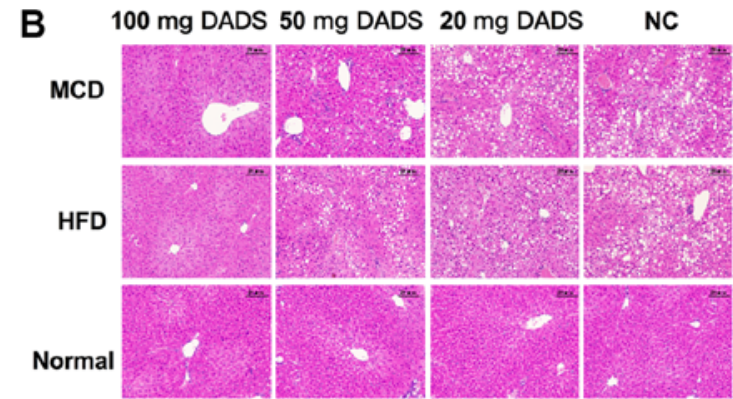

D

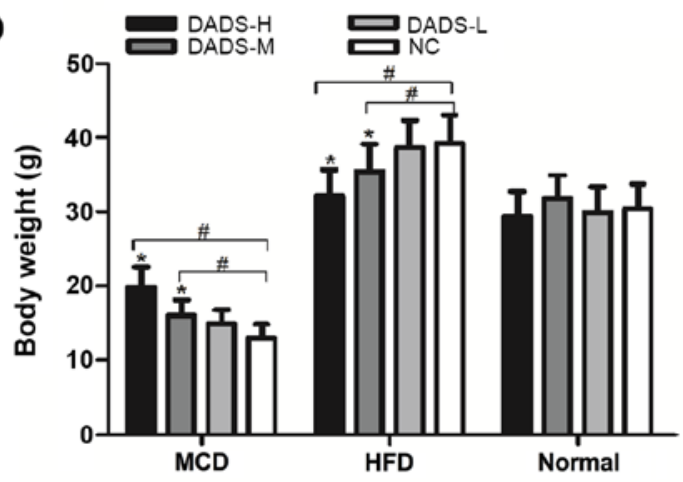

$\mathbf{F}$
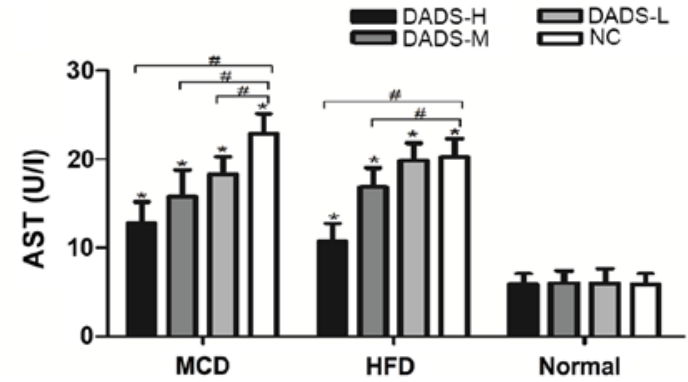

Figure 1. Evaluation of hepatic dysfunction in MCD-fed or HFD-fed mice with or without DADS treatment. (A) Representative images of liver morphology and (B) hematoxylin and eosin-stained liver sections (scale bar, $20 \mu \mathrm{m}$ ). (C) Fresh liver weights in the mice. (D) Body weights in the mice. (E) ALT level and (F) AST levels in plasma. Data were presented as mean \pm standard error of the mean; $n=5$. $\mathrm{P}<0.05$ vs. normal group with same treatment; ${ }^{\#} \mathrm{P}<0.05$ vs. NC in the same models. MCD, methionine- and choline-deficient diet; HFD, high-fat diet; DADS, diallyl disulfide; ALT, alanine transferase; AST, aspartate amino transferase; NC, negative control.

Inc.), and statistical significance was calculated using one-way analysis of variance. Post hoc multiple comparison testing among groups was performed using the least significance difference test. All experiments were repeated independently a minimum of three times. $\mathrm{P}<0.05$ was considered to indicate a statistically significant difference.

\section{Results}

DADS ameliorated hepatic dysfunction in mouse models of $N A S H$. Following 4 weeks of feeding with MCD or 20 weeks of feeding with HFD, the liver morphology and liver sections in the mice exhibited significant vacuolated hepatocytes, marked inflammatory cell infiltration and severe micro- and macro-vesicular steatosis, which indicated the successful establishment of two NASH mouse models (Fig. 1A and B). The inclusion of DADS (50 and $100 \mathrm{mg}$ ) in the MCD or HFD models, respectively, greatly ameliorated the hepatic dysfunction, as determined via H\&E staining (Fig. 1B). It is worth noting that supplementation with DADS had no effect on liver histology in mice fed a normal diet, demonstrating its non-hepatotoxic properties (P>0.05; Fig. 1B, E and F). Mice fed with MCD had the lowest liver weight and mice fed with HFD had the heaviest liver weight compared with those mice fed with a normal diet $(\mathrm{P}<0.01$; Fig. 1C). DADS (50 and $100 \mathrm{mg}$ ) significantly increased the liver weight in MCD-fed mice and decreased it in HFD-fed mice, respectively $(\mathrm{P}<0.01$; Fig. 1C). The trend of changes in body weight was similar to the liver weight in mice $(\mathrm{P}<0.05$; Fig. 1D). Furthermore, the serum ALT and AST levels were significantly increased in these two NASH models, and these levels were significantly decreased by DADS treatment in a dose-dependent manner $(\mathrm{P}<0.05$; Fig. 1E and $\mathrm{F})$.

DADS decreased hepatic lipid accumulation in mouse models of NASH. Oil Red O staining was performed in liver sections in order to investigate the effect of DADS on lipid deposition and lipid droplet accumulation. As presented in Fig. 2A, the hepatic lipid accumulation was greater in mouse models of NASH than in the mice fed with a normal diet $(\mathrm{P}<0.05)$. 

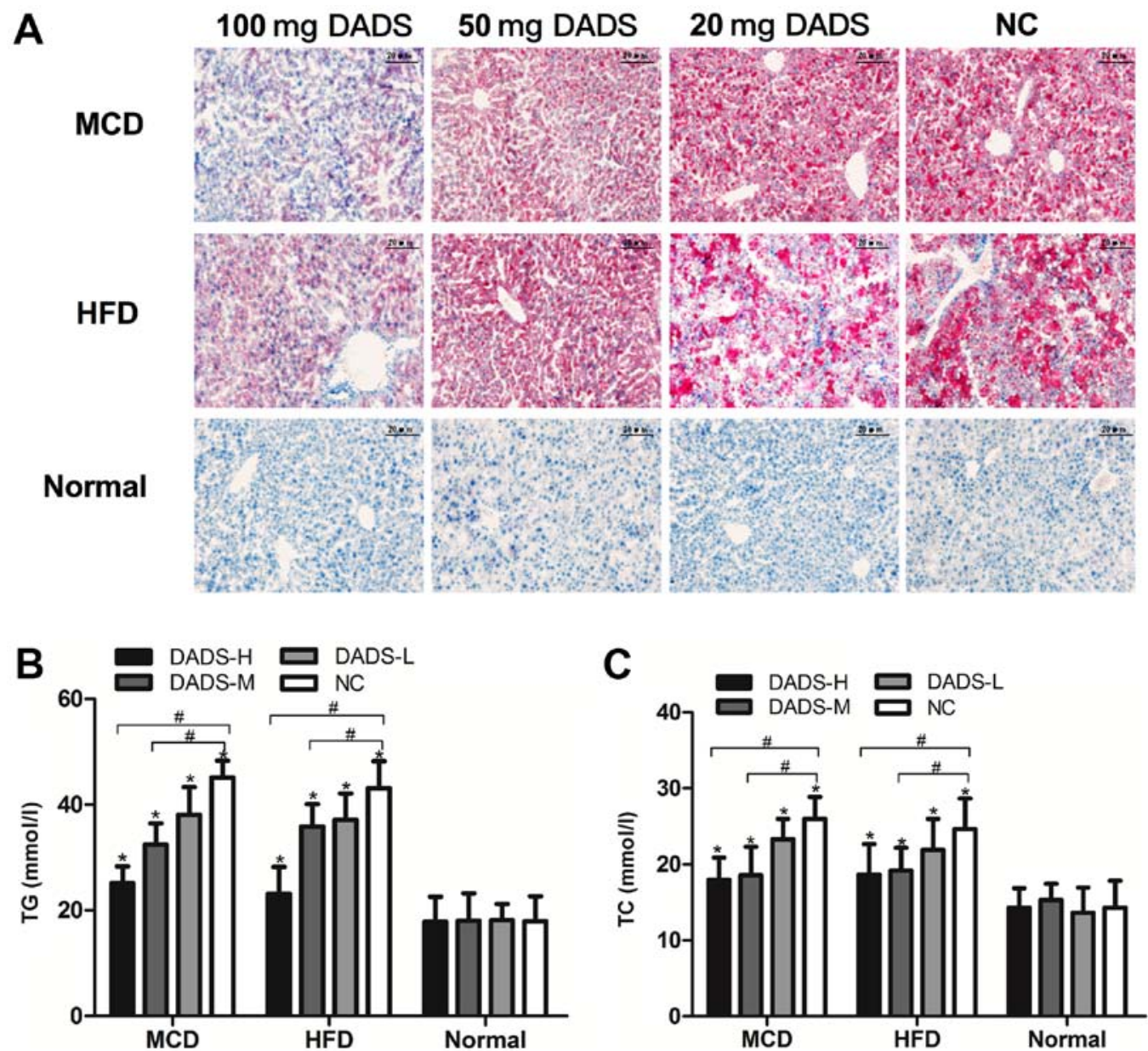

Figure 2. Hepatic lipid accumulation in MCD-fed or HFD-fed mice with or without DADS treatment. (A) Representative images of oil red O stained liver sections (scale bar, $20 \mu \mathrm{m}$ ). (B) The hepatic TG and (C) TC content were measured using GPO-PAP and COD-PAP enzymatic colorimetric assay kits. Data are presented as mean \pm standard error of the mean; $n=5$. $P<0.01$ vs. normal group with same treatment; ${ }^{*} \mathrm{P}<0.01$ vs. NC in the same models. MCD, methionine- and choline-deficient diet; HFD, high-fat diet; DADS, diallyl disulfide; TG, serum triglyceride; TC, total cholesterol; NC, negative control; NASH, non-alcoholic steatohepatitis.

DADS treatment caused a marked decrease in the number and size of lipid droplets $(\mathrm{P}<0.01 ;$ Fig. 2A). The liver TG and TC contents in MCD-fed mice were elevated by 2.51- and 1.81-fold in MCD-fed mice, respectively, compared with the mice fed a normal $\operatorname{diet}(\mathrm{P}<0.05$; Fig. $2 \mathrm{~B}$ and $\mathrm{C})$. These contents were also increased by 2.42 - and 1.72-fold in HFD-fed mice, respectively, when compared with those mice fed a normal $\operatorname{diet}(\mathrm{P}<0.05$; Fig. 2B and $\mathrm{C})$. Interestingly, DADS treatment significantly decreased MCD-induced or HFD-induced elevations in the liver TG and TC contents in a dose-dependent manner ( $\mathrm{P}<0.05$; Fig. $2 \mathrm{~B}$ and $\mathrm{C})$. These results clearly indicated the anti-steatotic role of DADS on MCD- or HFD-induced NASH. Since the high-dose DADS group displayed the best hepatoprotective effects, $100 \mathrm{mg} / \mathrm{kg}$ DADS was chosen for the following experiments.

DADS ameliorated hepatic steatosis by regulating key regulators of lipid metabolism. To investigate the molecular mechanisms behind DADS in ameliorating hepatic steatosis, the mRNA levels of key lipid metabolism-associated genes were examined. MCD or HFD caused a significant increase in the mRNA levels of SREBP-1c and apolipoprotein A1 (ApoA-I), while they led to a significant decrease in the
mRNA levels of CREBH and fibroblast growth factor (FGF)21 $(\mathrm{P}<0.05$; Fig. $3 \mathrm{~A}$ and $\mathrm{B})$. In addition, DADS treatment reversed the effect of MCD or HFD on the expression of these genes $(\mathrm{P}<0.05$; Fig. 3A and $\mathrm{B})$. As the major regulators of lipid biosynthesis, SREBP-1c and CREBH regulate the expression of several genes associated with TG and fatty acid (FA) synthesis. Thus, the protein levels of SREBP-1c and CREBH were detected and the results revealed that DADS decreased the protein expression levels of SREBP-1c and increased the protein expression levels of CREBH ( $\mathrm{P}<0.05$; Fig. $3 \mathrm{C}$ and $\mathrm{D})$.

DADS attenuated lipotoxicity and lipid peroxidation in $H F D$-fed mice. To investigate the role of DADS in lipotoxicity and lipid peroxidation in HFD-fed mice, the expression levels of PPAR $\alpha$ and SCD-1 were detected in the present study. As presented in Fig. 4A and B, the expression levels of PPAR $\alpha$ or SCD-1 were significantly decreased or increased, respectively, in HFD-fed mice when compared with the normal control group $(\mathrm{P}<0.05)$. DADS treatment led to an increase in PPAR $\alpha$ expression and a decrease in SCD-1 expression in HFD-fed mice ( $\mathrm{P}<0.05$; Fig. $4 \mathrm{~A}$ and $\mathrm{B})$. These results were further verified via an immunofluorescence assay, demonstrating the expression levels of PPAR $\alpha$ and SCD-1 (Fig. 4C). 

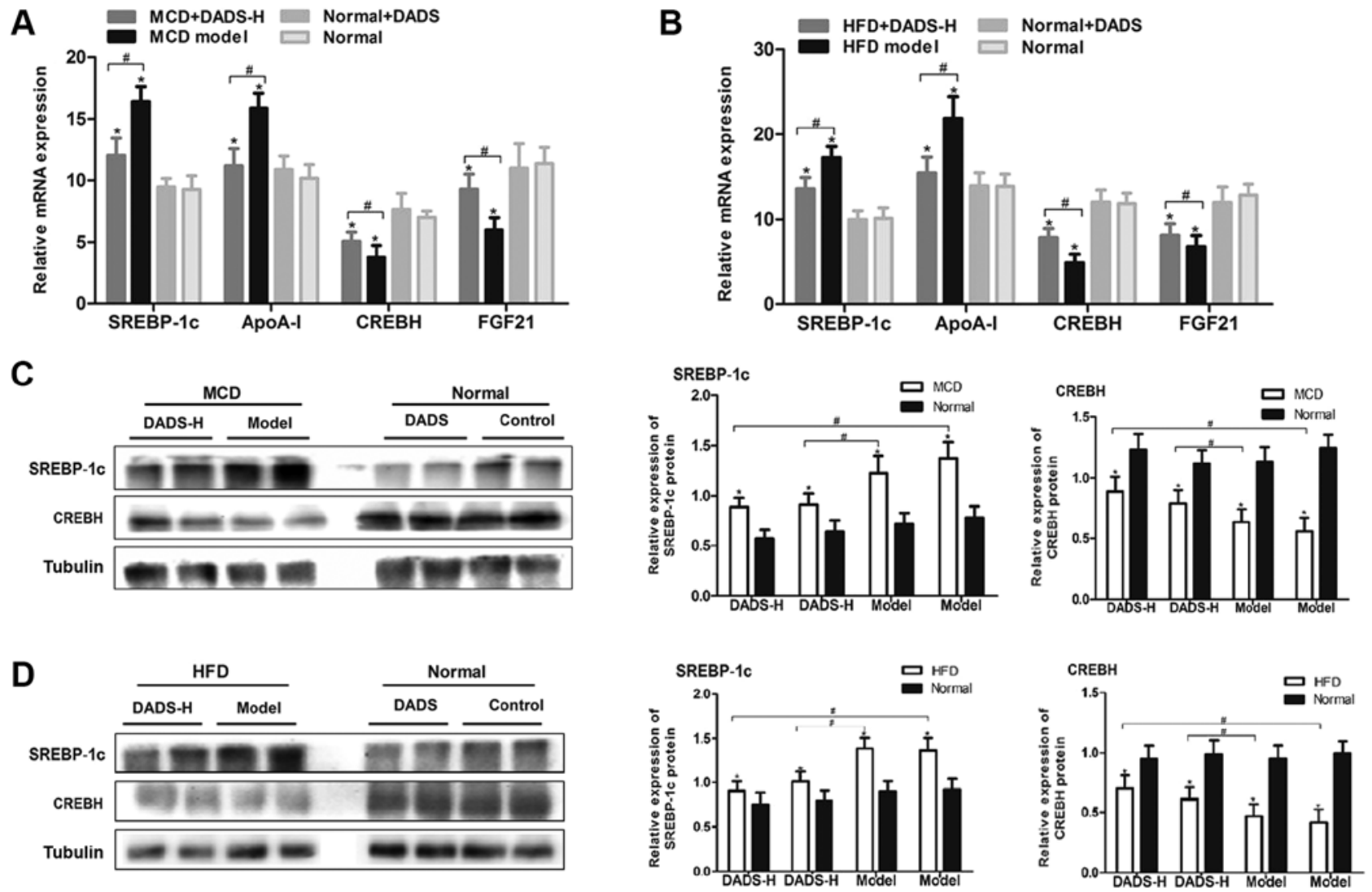

Figure 3. Effects of DADS on key molecules of lipid metabolism in NASH mice. (A and B) reverse transcription-quantitative PCR analyses of hepatic mRNA levels for critical genes involved in lipid metabolism in the MCD and HFD models. Specific mRNA expression values were normalized to the expression of $\beta$-actin. (C and D) Total protein levels of SREBP-1c, CREBH and tubulin were analyzed using western blotting in MCD models and HFD models. Tubulin was used as the internal control. Data are presented as mean \pm standard error of the mean; $n=5$. $P<0.01$ vs. normal group; ${ }^{*}<<0.05$ vs. NASH models. DADS, diallyl disulfide; NASH, non-alcoholic steatohepatitis; MCD, methionine- and choline-deficient diet; HFD, high-fat diet; SREBP-1c, sterol regulatory element-binding transcription factor 1; ApoA-I, apolipoprotein A1; CREBH, cyclic AMP-responsive element-binding protein H; FGF21, fibroblast growth factor 21.

The abnormalities in MDA and SOD levels in the plasma could also be alleviated by DADS treatment in HFD-fed mice $(\mathrm{P}<0.05$; Fig. 4D).

DADS inhibits inflammation through the $N F-\kappa B$ pathway in $M C D$-fed mice. The results of immunohistochemical staining of F4/80-positive cells revealed that the number of macrophage cells had increased in the liver in MCD-fed group, while it had decreased following DADS treatment $(\mathrm{P}<0.05$; Fig. 5A). TNF- $\alpha$ and IL-6 are the two most common inflammatory cytokines. TNF- $\alpha$ is the earliest and most important inflammatory mediator in the inflammatory process; it activates neutrophils and lymphocytes and allows them to pass through the vascular endothelial cells. In addition, TNF- $\alpha$ regulates other metabolic activities in the tissues and promotes the synthesis and release of other cytokines. IL- 6 induces B-cell differentiation and produces antibodies. It also induces the activation, proliferation and differentiation of sputum cells, and participates in the immune response of the body. IL-6 is a trigger for inflammatory reactions. Therefore, these two most representative inflammatory factors were chosen to assess the therapeutic effects of DADS in the present study. The plasma levels of IL- 6 and TNF- $\alpha$ were higher in the MCD-fed mice than in the mice that were fed a normal diet $(\mathrm{P}<0.05$; Fig. 5B). In addition, DADS treatment in MCD-fed mice effectively reversed these elevations $(\mathrm{P}<0.05$; Fig. $5 \mathrm{~B})$. We then detected the expression of the NF- $\kappa \mathrm{B}$ pathway, which was revealed to play a pivotal role in the pathogenesis of NASH. As presented in Fig. 5C and D, MCD led to an increase in the protein expression levels of $\mathrm{p}-\mathrm{NF}-\kappa \mathrm{B}$ p65 in the liver of MCD-fed mice, and DADS treatment blocked this increase $(\mathrm{P}<0.05)$.

\section{Discussion}

The global incidence of NASH increases constantly with the improvement of living standards. However, the medications that have been proven to be most effective for NASH are limited, and treatment for end-stage NASH is confined to liver transplantation only $(23,24)$. Thus, discovering effective drugs for $\mathrm{NASH}$ and elucidating its underlying molecular mechanism is urgently required. Herein, the results from the present study demonstrated the apparent hepatoprotective effect of DADS that was involved in regulating the key regulators of hepatic steatosis, lipotoxicity, lipid peroxidation and inflammation. Diversified animal models of NASH have been established to understand its etiology and to discover efficient therapies. Two mouse models of NASH induced by MCD or HFD were established, which were associated with decreased very-low-density lipoprotein secretion or increased FA levels associated 
A

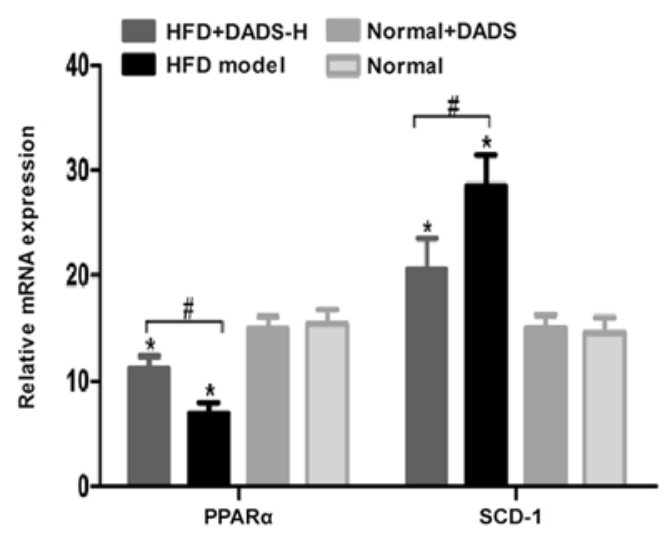

C

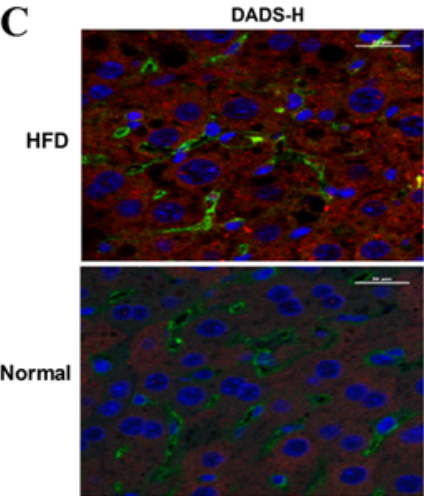

B
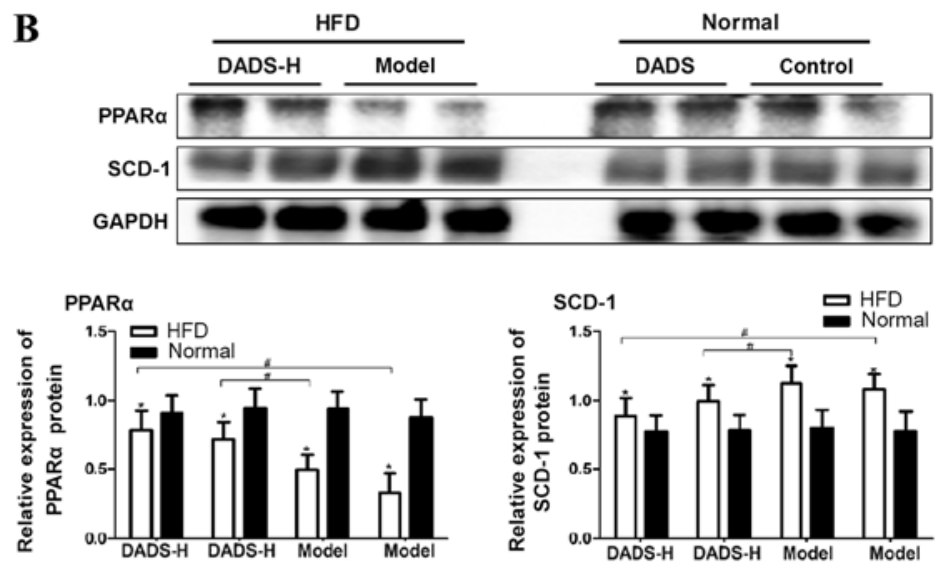

D
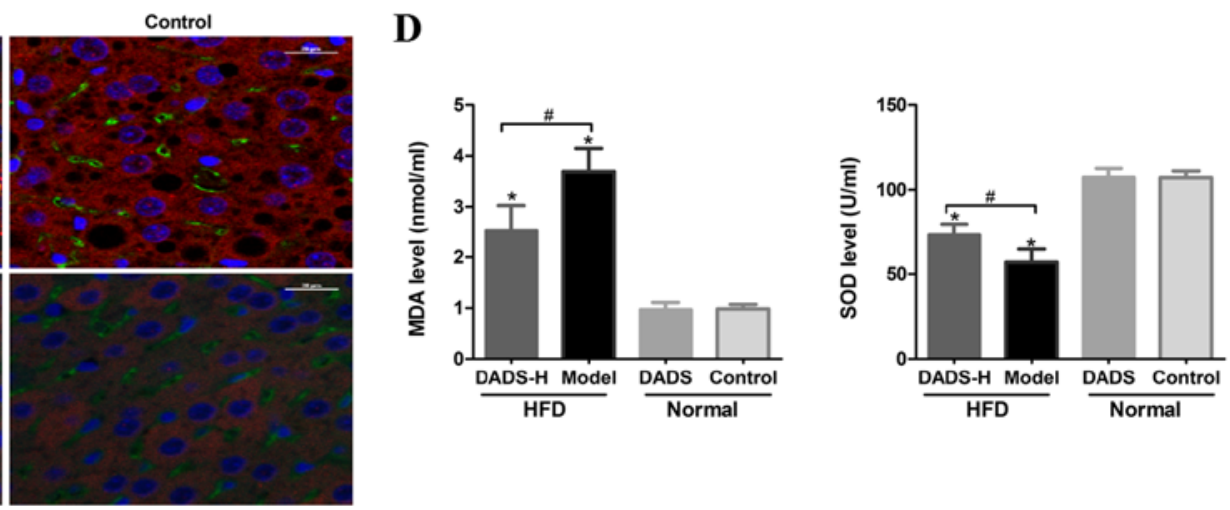

Figure 4. Lipotoxicity and lipid peroxidation in HFD-fed mice with or without DADS treatment. (A) Relative mRNA expression levels of PPAR $\alpha$ and SCD-1 were detected via reverse transcription quantitative-PCR. (B) Western blot analyses of hepatic protein levels of PPAR $\alpha$ and SCD-1 in HFD-fed mice. (C) Representative images of immunofluorescence. Liver sections were co-stained CREBH (green) with PPAR $\alpha$ (red) or SCD-1 (-red) and DAPI (blue). Three independent experiments were carried out. Scale bar: $20 \mu \mathrm{m}$. (D) The expression of MDA and SOD were examined by ELISA assay. Data were presented as mean $\pm \mathrm{SEM} ; \mathrm{n}=5$. ${ }^{*} \mathrm{P}<0.01$ vs. normal group; ${ }^{*} \mathrm{P}<0.05$ vs. HFD model group. HFD, high-fat diet; DADS, diallyl disulfide; PPAR $\alpha$, peroxisome proliferator-activated receptor $\alpha$; SCD-1, stearoyl-coenzyme A desaturase 1.

with the liver, respectively (25). Consistent with a previous study, the results from the present study revealed that both MCD and HFD induced the histological features of NASH in mice, and included significant vacuolated hepatocytes, marked inflammatory cell infiltration and severe micro- and macro-vesicular steatosis (Fig. 1A and B) (19). Mice fed with an MCD had the lowest liver weight when compared with that of the mice fed a normal diet (Fig. 1C). The trend of changes with body weight was similar to the liver weight in mice $(\mathrm{P}<0.05$; Fig. 1D). Furthermore, the serum ALT and AST levels, which are sensitive markers of liver damage, were also significantly increased in these two NASH models (Fig. 1E and F). In addition to the hepatic dysfunction, hepatic lipid accumulation was observed in the MCD-fed and HFD-fed mice (Fig. 2A). The two mouse models of NASH induced by MCD and HFD were associated with reduced very-low-density lipoprotein secretion and increased FA delivery to the liver, respectively. MCD and HFD induced the histological features of $\mathrm{NASH}$ in mice, including significant vacuolated hepatocytes, marked inflammatory cell infiltration and severe micro- and macro-vesicular steatosis. Previous studies have suggested that DADS has potential as a treatment option for metabolic syndrome and acute liver injury due to its hepatoprotective and antioxidant effects $(5,17)$. Thus, amelioration of hepatic dysfunction by
DADS was thought not to be due to direct interactions with either the MCD or HFD diet. Excessive TG and TC accumulation in the liver has been proven to be an early event in NASH, which may be attributed to the enhancement of FA synthesis and to the impairment of FA oxidation $(26,27)$. It was revealed that there were significantly greater liver TG and TC contents in MCD-fed and HFD-fed mice when compared with those mice fed a normal diet (Fig. 2B and C).

DADS is one of the most abundant, oil-soluble organosulfur compounds derived from garlic. It has been demonstrated to have protective effects against several diseases (9). In the present study, it was revealed that DADS treatment ameliorated hepatic dysfunction and reduced hepatic lipid accumulation in MCD-fed and HFD-fed mice (Figs. 1 and 2). Previous studies have also suggested that DADS demonstrated potential in the treatment of metabolic syndrome and acute liver injury $(5,17,28)$. Lee et al (28) revealed the hepatoprotective and antioxidant effects of DADS on carbon tetrachloride-induced hepatic oxidative damage and the inflammatory response in rats. To elucidate the underlying molecular mechanisms of DADS in ameliorating hepatic steatosis, the expression of key lipid metabolism-associated genes was examined. It was revealed that DADS decreased the expression levels of SREBP-1c and ApoA-I and increased the expression levels of CREBH and FGF21 (Fig. 3A and B). Lipid metabolism-associated SREBP-1c 
A

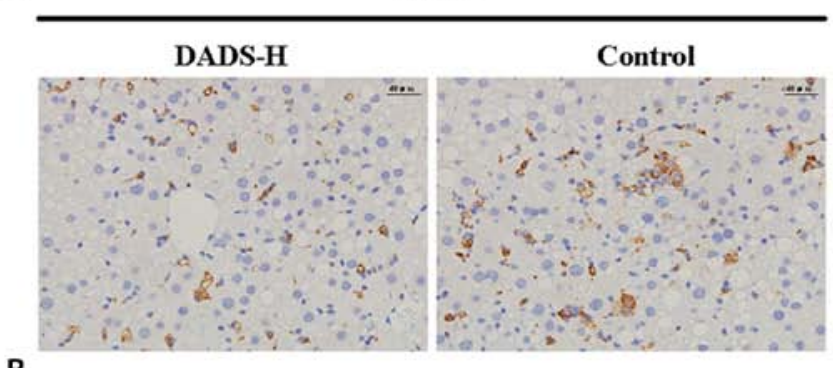

B

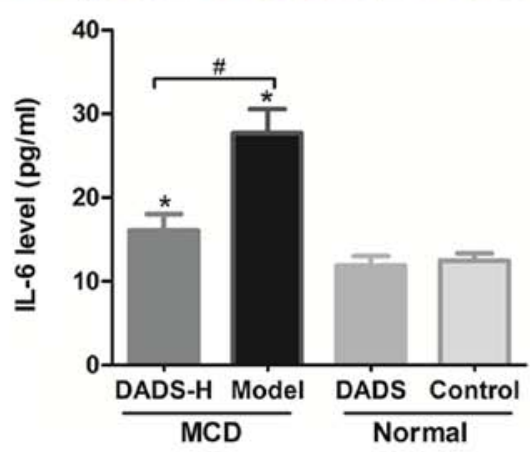

C

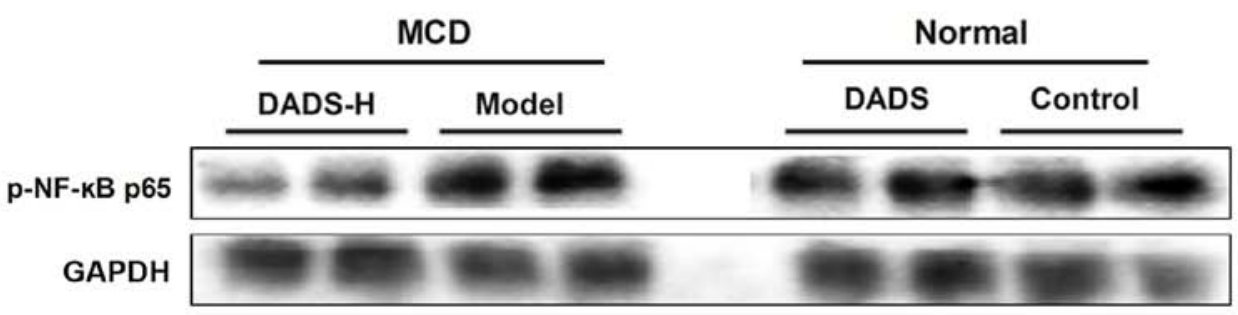

D

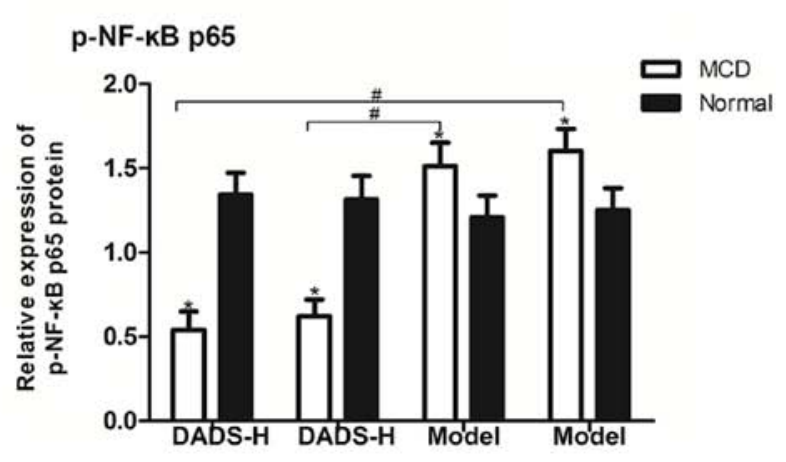

Figure 5. DADS inhibited inflammation through NF- $\kappa$ B pathway in MCD-fed mice. (A) Representative images of immunohistochemistry. The expression of F4/80 was detected in liver sections (scale bar: $40 \mu \mathrm{m}$ ). (B) The expression of TNF- $\alpha$ and IL-6 were examined by ELISA assay. (C and D) Total protein levels of p-NF- $\mathrm{B}$ p65 and GAPDH were analyzed using western blotting in MCD models. GAPDH was used as the internal control. Data were presented as mean \pm standard error of the mean; $\mathrm{n}=5$. ${ }^{*} \mathrm{P}<0.01$ vs. normal control group; ${ }^{*} \mathrm{P}<0.01$ vs. MCD model group. DADS, diallyl disulfide; $\mathrm{NF}-\kappa \mathrm{B}$, nuclear factor- $\kappa \mathrm{B}$; TNF, tumor necrosis factor; IL, interleukin; MCD, methionine- and choline-deficient diet.

has been indicated to regulate several crucial genes associated with TG synthesis, including ApoA-I and SCD-1, thus playing a pivotal role in hepatic steatosis (29). Furthermore, CREBH can modulate the expression levels of genes involved in lipogenesis, sterol metabolism, lipid peroxidation, lipotoxicity, inflammation and gluconeogenesis $(30,31)$. These data indicated that DADS ameliorated hepatic steatosis by regulating SREBP-1c and CREBH, the key regulators of lipid metabolism (Fig. 3C and D).

In addition, data from the present study also revealed that the expression levels of the genes involved in FA oxidation (including PPAR $\alpha$ and SCD-1) and lipid peroxidation (including MDA and SOD) were reversed by DADS in HFD-fed mice (Fig. 4). Decreased PPAR $\alpha$ expression may result in the reduction of FA oxidation and lead to an acceleration of lipid accumulation in the liver, thereby contributing to the development of NASH. MDA is a marker for oxidative stress, which can lead to lipid peroxidation. The anti-oxidant enzyme SOD is capable of eliminating ROS, which leads to hepatic apoptosis. Therefore, upregulated PPAR $\alpha$, decreased MDA and increased SOD induced by DADS indicated its attenuated effect on the lipotoxicity and lipid peroxidation in HFD-fed mice.

In addition to lipid peroxidation, the inflammatory response was also a striking feature of NASH. The inflammatory response in the NASH model induced by MCD was investigated in the present study, which could induce high-grade hepatic inflammatory cell infiltration. It was revealed that DADS dramatically 
reduced the number of macrophage cells in the liver (Fig. 5A). Furthermore, DADS also decreased the expression levels of TNF- $\alpha$ and IL-6 (Fig. 5B), which is consistent with results from previous studies $(32,33)$. The activity of $\mathrm{NF}-\kappa \mathrm{B}$ was decreased by DADS treatment in MCD-fed mice (Fig. $5 \mathrm{C}$ and D). It has been reported that DADS was effective in reducing the levels of oxidized low-density lipoprotein, lipid peroxidation, as well as NF- $\kappa \mathrm{B}$ activity, revealing good anti-inflammatory and antioxidant properties (34). The results from the present study demonstrated that the therapeutic effects of DADS on NASH were associated with decreased inflammatory cell infiltration and downregulated pro-inflammatory cytokines in MCD-fed mice.

There were certain limitations to the present study. The results of the study indicated that DADS exerted beneficial effects on MCD- or HFD-induced NASH by suppressing key regulators of lipid metabolism, lipid peroxidation and inflammation; however, insulin resistance and abnormal sterol metabolism are also involved in the pathogenesis of NASH. Whether DADS improves insulin resistance and sterol metabolism abnormalities requires further investigation. Previous research has demonstrated that DADS inhibited the proliferation and transdifferentiation of lung fibroblasts via the induction of cyclooxygenase and the synthesis of prostaglandin $\mathrm{E}_{2}(35)$, suggesting that DADS may also have an important role in the pathogenesis of hepatic fibrosis. It will serve as the direction for future research.

In conclusion, DADS effectively attenuated hepatic steatosis, lipotoxicity, lipid peroxidation and inflammation in NASH mouse models. These results suggest that DADS may be a potential therapeutic agent in the prevention and treatment of $\mathrm{NASH}$, given its high efficacy and low risk of side effects.

\section{Acknowledgements}

Not applicable.

\section{Funding}

The present study was supported by the National Natural Science Foundation of China (grant no. 81670515).

\section{Availability of data and materials}

The datasets used and/or analyzed during the present study are available from the corresponding author upon reasonable request.

\section{Authors' contributions}

NZ and YW conceived and designed the study. NZ, JZ, BL, SX and GL performed the experiments. NZ and YW analyzed the data and drafted the manuscript. KX conceived the study and gave final approval of the version to be published. All authors read and approved the manuscript.

\section{Ethics approval and consent to participate}

The experimental protocol was approved by the Animal Ethics Committee of Tongji Medical College, Huazhong University of Science and Technology (Wuhan, China), and performed according to the principles of the Institutional Animal Care and Ethics Committee guidelines (IACUC no. S619).

\section{Patient consent for publication}

Not applicable.

\section{Competing interests}

The authors declare that they have no competing interests.

\section{References}

1. Italian Association for the Study of the Liver (AISF): AISF position paper on nonalcoholic fatty liver disease (NAFLD): Updates and future directions. Dig Liver Dis 49: 471-483, 2017.

2. Caligiuri A, Gentilini A and Marra F: Molecular pathogenesis of NASH. Int J Mol Sci 17: pii: E1575, 2016.

3. Tilg $\mathrm{H}$ and Moschen AR: Evolution of inflammation in nonalcoholic fatty liver disease: The multiple parallel hits hypothesis. Hepatology 52: 1836-1846, 2010.

4. Lassailly G, Caiazzo R, Pattou F and Mathurin P: Perspectives on treatment for nonalcoholic steatohepatitis. Gastroenterology 150: 1835-1848, 2016.

5. Lai YS, Chen WC, Ho CT, Lu KH, Lin SH, Tseng HC, Lin SY and Sheen LY: Garlic essential oil protects against obesity-triggered nonalcoholic fatty liver disease through modulation of lipid metabolism and oxidative stress. J Agric Food Chem 62: 5897-5906, 2014.

6. Yun HM, Ban JO, Park KR, Lee CK, Jeong HS, Han SB and Hong JT: Potential therapeutic effects of functionally active compounds isolated from garlic. Pharmacol Ther 142: 183-195, 2014.

7. Sheen LY, Wu CC, Lii CK and Tsai SJ: Effect of diallyl sulfide and diallyl disulfide, the active principles of garlic, on the aflatoxin $\mathrm{B}(1)$-induced DNA damage in primary rat hepatocytes. Toxicol Lett 122: 45-52, 2001

8. Liu Y, Li A, Feng X, Sun X, Zhu X and Zhao Z: Pharmacological investigation of the anti-inflammation and anti-oxidation activities of diallyl disulfide in a rat emphysema model induced by cigarette smoke extract. Nutrients 10: pii: E79, 2018.

9. Ko JW, Park SH, Shin NR, Shin JY, Kim JW, Shin IS, Moon C, Heo JD, Kim JC and Lee IC: Protective effect and mechanism of action of diallyl disulfide against acetaminophen-induced acute hepatotoxicity. Food Chem Toxicol 109: 28-37, 2017.

10. Raghu R, Liu CT, Tsai MH, Tang X, Kalari KR, Subramanian S and Sheen LY: Transcriptome analysis of garlic-induced hepatoprotection against alcoholic fatty liver. J Agric Food Chem 60: 11104-11119, 2012.

11. Shimada M, Liu L, Nussler N, Jonas S, Langrehr JM, Ogawa T, Kaminishi M, Neuhaus P and Nussler AK: Human hepatocytes are protected from ethanol-induced cytotoxicity by DADS via CYP2E1 inhibition. Toxicol Lett 163: 242-249, 2006.

12. Jeong HG and Lee YW: Protective effects of diallyl sulfide on $\mathrm{N}$-nitrosodimethylamine-induced immunosuppression in mice. Cancer Lett 134: 73-79, 1998.

13. Guyonnet D, Siess MH, Le Bon AM and Suschetet M: Modulation of phase II enzymes by organosulfur compounds from allium vegetables in rat tissues. Toxicol Appl Pharmacol 154: 50-58, 1999.

14. Feng C, Luo Y, Nian Y, Liu D, Yin X, Wu J, Di J, Zhang R and Zhang J: Diallyl disulfide suppresses the inflammation and apoptosis resistance induced by DCA through ROS and the NF- $\kappa$ B signaling pathway in human barrett's epithelial cells. Inflammation 40: 818-831, 2017.

15. Khatua TN, Dinda AK, Putcha UK and Banerjee SK: Diallyl disulfide ameliorates isoproterenol induced cardiac hypertrophy activating mitochondrial biogenesis via eNOS-Nrf2-Tfam pathway in rats. Biochem Biophys Rep 5: 77-88, 2015.

16. Battal M, Kartal A, Citgez B, Yilmaz B, Akcakaya A and Karatepe O: Impact of allyl disulfide on oxidative damage and liver regeneration in an experimental hepatectomy model. Chirurgia (Bucur) 110: 117-122, 2015.

17. Lee IC, Kim SH, Baek HS, Moon C, Kim SH, Kim YB, Yun WK, Kim HC and Kim JC: Protective effects of diallyl disulfide on carbon tetrachloride-induced hepatotoxicity through activation of Nrf2. Environ Toxicol 30: 538-548, 2015. 
18. Germain E, Chevalier J, Siess MH and Teyssier C: Hepatic metabolism of diallyl disulphide in rat and man. Xenobiotica 33: 1185-1199, 2003

19. Fan JG and Qiao L: Commonly used animal models of non-alcoholic steatohepatitis. Hepatobiliary Pancreat Dis Int 8: 233-240, 2009.

20. Zhang J, Zhang H, Deng X, Zhang N, Liu B, Xin S, Li G and $\mathrm{Xu} \mathrm{K}$ : Baicalin attenuates non-alcoholic steatohepatitis by suppressing key regulators of lipid metabolism, inflammation and fibrosis in mice. Life Sci 192: 46-54, 2018.

21. Kleiner DE, Brunt EM, Van Natta M, Behling C, Contos MJ, Cummings OW, Ferrell LD, Liu YC, Torbenson MS Unalp-Arida A, et al: Design and validation of a histological scoring system for nonalcoholic fatty liver disease. Hepatology 41 : 1313-1321, 2005.

22. Livak KJ and Schmittgen TD: Analysis of relative gene expression data using real-time quantitative PCR and the 2(-Delta Delta C(T)) method. Methods 25: 402-408, 2001.

23. Bellentani S: The epidemiology of non-alcoholic fatty liver disease. Liver Int 37 (Suppl 1): S81-S84, 2017.

24. Musso G, Cassader M and Gambino R: Non-alcoholic steatohepatitis: Emerging molecular targets and therapeutic strategies. Nat Rev Drug Discov 15: 249-274, 2016.

25. Imajo K, Yoneda M, Kessoku T, Ogawa Y, Maeda S, Sumida Y, Hyogo H, Eguchi Y, Wada K and Nakajima A: Rodent models of nonalcoholic fatty liver disease/nonalcoholic steatohepatitis. Int J Mol Sci 14: 21833-21857, 2013.

26. Banini BA and Sanyal AJ: Nonalcoholic fatty liver disease: Epidemiology, pathogenesis, natural history, diagnosis, and current treatment options. Clin Med Insights Ther 8: 75-84, 2016

27. Benedict $\mathrm{M}$ and Zhang X: Non-alcoholic fatty liver disease: An expanded review. World J Hepatol 9: 715-732, 2017.
28. Lee IC, Kim SH, Baek HS, Moon C, Kang SS, Kim SH, Kim YB, Shin IS and Kim JC: The involvement of Nrf2 in the protective effects of diallyl disulfide on carbon tetrachloride-induced hepatic oxidative damage and inflammatory response in rats. Food Chem Toxicol 63: 174-185, 2014.

29. Nagaya T, Tanaka N, Suzuki T, Sano K, Horiuchi A, Komatsu M, Nakajima T, Nishizawa T, Joshita S, Umemura T, et al: Down-regulation of SREBP-1c is associated with the development of burned-out NASH. J Hepatol 53: 724-731, 2010.

30. Del Campo JA, Gallego-Durán R, Gallego P and Grande L: Genetic and epigenetic regulation in nonalcoholic fatty liver disease (NAFLD). Int J Mol Sci 19: pii: E911, 2018.

31. Lee JH, Giannikopoulos P, Duncan SA, Wang J, Johansen CT, Brown JD, Plutzky J, Hegele RA, Glimcher LH and Lee AH: The transcription factor cyclic AMP-responsive element-binding protein $\mathrm{H}$ regulates triglyceride metabolism. Nat Med 17: 812-815, 2011.

32. Lee IC, Baek HS, Kim SH, Moon C, Park SH, Kim SH, Shin IS, Park SC and Kim JC: Effect of diallyl disulfide on acute gastric mucosal damage induced by alcohol in rats. Hum Exp Toxicol 34: 227-239, 2015.

33. Bauer D, Mazzio E, Soliman KF, Taka E, Oriaku E, Womble T and Darling-Reed S: Diallyl disulfide inhibits TNFo-induced CCL2 release by MDA-MB-231 cells. Anticancer Res 34: 2763-2770, 2014

34. Rai SK, Sharma M and Tiwari M: Inhibitory effect of novel diallyldisulfide analogs on HMG-CoA reductase expression in hypercholesterolemic rats: CREB as a potential upstream target. Life Sci 85: 211-219, 2009.

35. Wang Y, Cao R, Wei B, Chai X, Sun D, Guan Y and Liu XM: Diallyl disulfide inhibits proliferation and transdifferentiation of lung fibroblasts through induction of cyclooxygenase and synthesis of prostaglandin $\mathrm{E}_{2}$. Mol Cell Biochem 393: 77-87, 2014. 